\title{
Fully inverted single-digit nanometer domains in ferroelectric films
}

\author{
Noureddine Tayebi, ${ }^{1,2}$ Yoshie Narui, ${ }^{3}$ Nathan Franklin, ${ }^{1}$ C. Patrick Collier, ${ }^{3, a)}$ \\ Konstantinos P. Giapis, ${ }^{3}$ Yoshio Nishi, ${ }^{2}$ and Yuegang Zhang ${ }^{1,4, b)}$ \\ ${ }^{1}$ Intel Corporation, 2200 Mission College Boulevard, Santa Clara, California 95054, USA \\ ${ }^{2}$ Department of Electrical Engineering, Stanford University, 420 Via Palou Mall, Stanford, \\ California 94305, USA \\ ${ }^{3}$ Division of Chemistry and Chemical Engineering, California Institute of Technology, Pasadena, \\ California 91125, USA \\ ${ }^{4}$ The Molecular Foundry, Lawrence Berkeley National Laboratory, Berkeley, California 94720, USA
}

(Received 6 November 2009; accepted 5 December 2009; published online 12 January 2010)

\begin{abstract}
Achieving stable single-digit nanometer inverted domains in ferroelectric thin films is a fundamental issue that has remained a bottleneck for the development of ultrahigh density ( $>1$ Tbit/in. $\left.{ }^{2}\right)$ probe-based memory devices using ferroelectric media. Here, we demonstrate that such domains remain stable only if they are fully inverted through the entire ferroelectric film thickness, which is dependent on a critical ratio of electrode size to the film thickness. This understanding enables the formation of stable domains as small as $4 \mathrm{~nm}$ in diameter, corresponding to 10 unit cells in size. Such domain size corresponds to 40 Tbit/in. ${ }^{2}$ data storage densities. (C) 2010 American Institute of Physics. [doi:10.1063/1.3280371]
\end{abstract}

Probe-based seek-and-scan data storage systems based on ferroelectric thin films are pursued for ultrahigh density nonvolatile memory devices. ${ }^{1-7}$ Data recording in such systems is based on a short electric pulse applied through a conductive probe (or an array of probes), which creates a highly concentrated electric field that inverts the polarization of a local film volume, thus resulting in a nonvolatile ferroelectric domain. For storage density exceeding $1 \mathrm{Tbit} / \mathrm{in}^{2}$, domain size reduction below $10 \mathrm{~nm}$ is required.

Small domain sizes can be obtained by decreasing the size of the probe tip. Unfortunately, the inverted domain is subjected to ferroelectric depolarization charges and domain wall energy ${ }^{8-10}$ that can be high enough to invert the domain back to its initial polarization. It has been predicted ${ }^{9}$ that inverted ferroelectric domains smaller than $15 \mathrm{~nm}$ are unstable and could be inverted back to their initial state as soon as the electric pulse is removed. This instability can be further exacerbated by the presence of a built-in electric field due to film defects present in thin ferroelectric films. Here, we demonstrate that single-digit nanometer domains are stable if a critical ratio between probe size and ferroelectric film thickness is reached for a full polarization inversion. Using "nanopencil" probes, ${ }^{7}$ we were able to write stable domains only $4 \mathrm{~nm}$ in diameter in $17 \mathrm{~nm}$-thick ferroelectric films of $\mathrm{Pb}\left(\mathrm{Zr}_{1-\mathrm{x}} \mathrm{Ti}_{\mathrm{x}}\right) \mathrm{O}_{3} \quad$ (PZT) - corresponding to $40 \mathrm{Tbit} /$ in. $^{2}$ data storage densities.

Sub-10 nm inverted domains can be obtained using single-walled carbon nanotube (SWNT) probe tips. However, such tips are prone to bending and buckling when operating in contact mode. ${ }^{11,12}$ In the present study, we use dielectric sheathed SWNT probes, called nanopencils, which can operate in contact mode while withstanding forces as high as $14.5 \mu \mathrm{N}$ without bending and buckling. ${ }^{7}$ The dielectric sheath is a conformal coating of (65-nm-thick) $\mathrm{SiO}_{\mathrm{x}}$,

\footnotetext{
${ }^{a)}$ Present address: Oak Ridge National Laboratory, Center for Nanophase Materials Sciences, Oak Ridge, TN 37831, USA.

b) Author to whom correspondence should be addressed. Electronic mail: yzhang5@lbl.gov.
}

which also provides electrical insulation and exceptional wear characteristics. The fabrication of the nanopencil probes has been described elsewhere. ${ }^{7,13}$ Figures 1(a) and 1(b) show transmission electron microscopy (TEM) images of two nanopencil probes. The SWNT electrodes are exposed after a sharpening process on a conductive diamond film. ${ }^{7,13}$ The exposure is confirmed by conductive atomic force microscopy (AFM). Figures 1(c) and 1(d) show height images, current distribution maps, and I-V curves taken on the diamond film after electrode exposure. Both probes show very similar electrical characteristics with resistances of $3.6 \pm 0.5$ and $3.0 \pm .5 \mathrm{M} \Omega$ for the 9 and $3 \mathrm{~nm}$ SWNT electrodes, respectively.

The nanopencils are used to write inverted domain dots on an atomically smooth, single-crystal 50-nm-thick PZT film grown on $\mathrm{SrRuO}_{3} / \mathrm{SrTiO}_{3}(100)$ substrate using metalorganic chemical vapor deposition. ${ }^{13,14}$ The film is initially polarized in an upward direction. Figure 1(e) shows piezoresponse force microscopy (PFM) images of a $4 \times 1$ matrix of inverted dots written using the $9 \mathrm{~nm}$ SWNT electrode shown in Fig. 1(a) by applying $100 \mu$ s wide pulses of $7 \mathrm{~V}$, which is the smallest bias at which inverted domains could be detected. Dot sizes as small as $11.8 \mathrm{~nm}$ were reliably written at this bias [Fig. 1(e)]. When trying to write the dots using the $3 \mathrm{~nm}$ electrode shown in Fig. 1(b), however, no domain inversion was recorded even at $10 \mathrm{~V}$ [Fig. 1(f)].

In order to understand the origin of this discrepancy, we first characterized the ferroelectric properties of the PZT film using conventional capacitor testing methods. ${ }^{13}$ Very good butterflylike capacitance variation, squarelike hysteresis loop with high switching speeds and low leakage current $(2.75$ $\times 10^{-7} \mathrm{~A} / \mathrm{cm}^{2}$ ) are observed [Fig. 2(a) and 2(b)]. However, a large positive voltage shift is observed in both the capacitance and polarization variations, which indicates a 0.96 $\times 10^{7} \mathrm{~V} / \mathrm{m}$ built-in electric field $\left(E_{b}\right)$ [Fig. 2(b)]. This field is due to a remnant polarization that originates from near surface concentration of negative trapped charges of lead vacancies ${ }^{15}$ and is antiparallel to the inverted domain polarization. From the $\mathrm{C}-\mathrm{V}$ curve, the film has a dielectric con- 
(a)

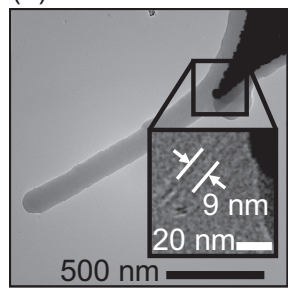

(c)

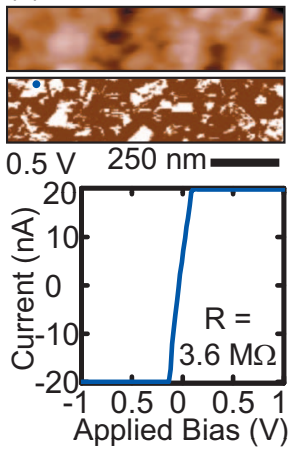

(e)

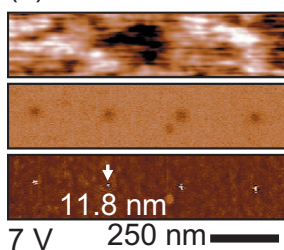

(b)

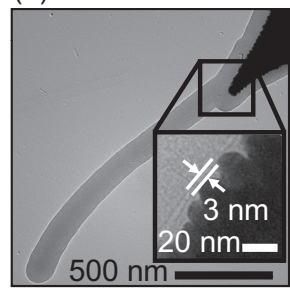

(d)
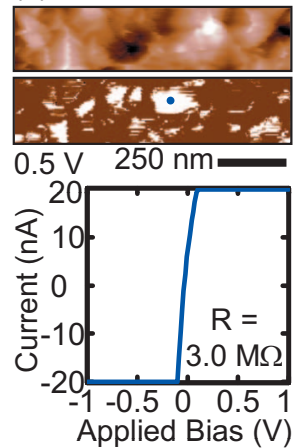

(f)

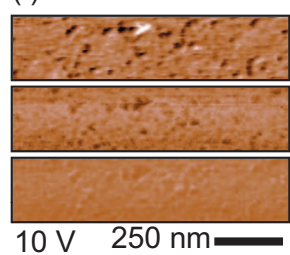

FIG. 1. (Color) [(a) and (b)] TEM images of nanopencil probes composed of a bundle of a few SWNTs with $9 \mathrm{~nm}$ overall diameter (a) and an individual SWNT with $3 \mathrm{~nm}$ diameter (b). [(c) and (d)] Conductive AFM height images (top), current distribution maps (middle) of a diamond film, and current-voltage (I-V) curves (bottom) taken after SWNT electrode exposures of (a) and (b), respectively. Blue dots in the current maps correspond to locations where I-V curves were taken. (e) PFM height (top), amplitude (middle), and phase (bottom) images of a 50-nm-thick PZT-film surface with $11.8 \mathrm{~nm}$ ferroelectric inverted domains formed by applying $7 \mathrm{~V}$ pulses to the film through the nanopencil shown in (a). (f) PFM images of the same PZT Film using the nanopencil shown in (b). No inverted domains are observed after $10 \mathrm{~V}$ pulses were applied.

stant $\varepsilon_{r}=95.6$ and a coercive field $E_{c}=1.70 \times 10^{7} \mathrm{~V} / \mathrm{m}$.

The obtained film properties are used to simulate the domain formation in the $50 \mathrm{~nm}$ PZT film by the nanopencil electrodes [Figs. 2(c) and 2(d)]. ${ }^{13}$ We can see that the $9 \mathrm{~nm}$ electrode creates a concentrated electric field underneath it that is high enough to form a fully inverted polarization domain through the entire film thickness down to the grounded electrode [Fig. 2(c)]. Note also that the lateral size of the written domain is expected to be larger than the actual tip size, which is also seen experimentally [Fig. 1(e)]. On the other hand, only partial inversion switching occurs for $3 \mathrm{~nm}$ probe [Fig. 2(d)]. As analyzed in the following paragraph, the partially inverted domain will be immediately inverted back after the removal of the writing pulse.

For a spherical domain, which is the case here [Fig. 2(d)], the total energy is given by ${ }^{9}$

$$
\Delta T=\frac{4 \pi}{3} a^{3}\left[\frac{2}{3} \frac{P_{s}^{2}}{\varepsilon_{r} \varepsilon_{o}}-2\left(E_{c}+E_{b}\right) P_{s}\right]+4 \pi a^{2} \gamma,
$$

where $a$ is the radius of the spherical domain, $P_{s}$ is the spontaneous polarization, $E_{c}$ is the coercive field, $E_{b}$ is the built-in bias (opposite sign of $E_{c}$ ), and $\gamma$ is the surface energy per unit area of the wall. The first term corresponds to the

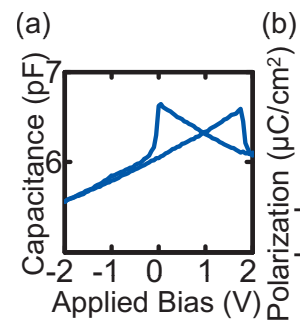

(c)
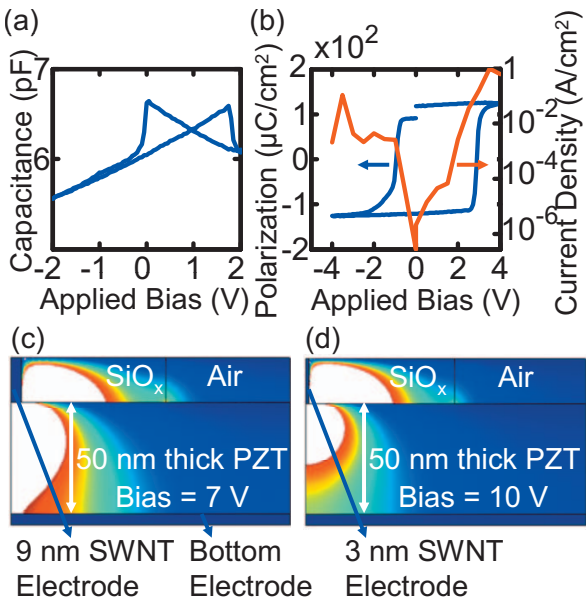

(d)

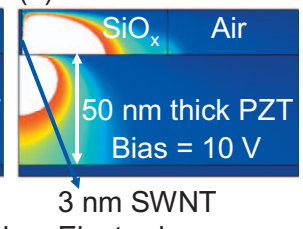

\section{Electrode}

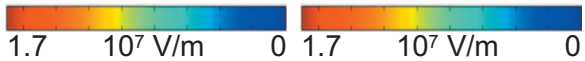

(e)

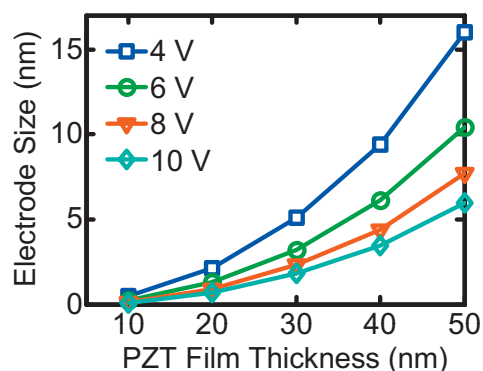

FIG. 2. (Color) [(a) and (b)] Capacitance-voltage hysteresis loop (a), polarization-voltage hysteresis loop and current-voltage variation (b). [(c) and (d)] Simulated cross-sectional mappings of the electric field component along the polarization axis under the same bias conditions of Figs. 1(e) and 1(f), i.e., $7 \mathrm{~V}$ for the $9 \mathrm{~nm} \mathrm{SWNT}$ electrode (c) and $10 \mathrm{~V}$ for the $3 \mathrm{~nm}$ electrode (d). Due to the axial symmetry, only half of the system is shown. The white areas correspond to electric field exceeding the experimental coercive field and are a measure of inverted domain volumes. (e) Minimum electrode size for full-thickness domain inversions for various PZT thicknesses at different applied biases.

change in electrostatic energy due to the introduction of the inverted domain, which includes the depolarization energy inside the domain and the energy induced by the coercive electric field. The second term is the surface energy of the domain wall. For the inverted domain to remain stable, the free energy reduction rate associated with the spherical domain, $f=-(\partial \Delta T) /(\partial a)$, has to be positive, i.e.,

$$
\begin{aligned}
f & =-\frac{\partial \Delta T}{\partial a}=-4 \pi a^{2}\left[\frac{2}{3} \frac{P_{s}^{2}}{\varepsilon_{r} \varepsilon_{o}}-2\left(E_{c}+E_{b}\right) P_{s}\right]-8 \pi a \gamma \\
& >0 .
\end{aligned}
$$

From Fig. 2(d), the radius of the spherical domain using the $3 \mathrm{~nm}$ SWNT electrode is estimated to be $18 \mathrm{~nm}$, whereas from Fig. 2(b) $P_{s}=124.6 \mu \mathrm{C} / \mathrm{cm}^{2}$. As indicated previously, $E_{c}=1.7 \times 10^{7} \mathrm{~V} / \mathrm{m}$ and $E_{b}=-0.96 \times 10^{7} \mathrm{~V} / \mathrm{m}$. The surface energy per unit area is taken to be $4 \mathrm{~mJ} / \mathrm{m}^{2}$ (Ref. 8). Given these values, the energy reduction rate is $f=-5.35 \mu \mathrm{J} / \mathrm{m}$, indicating that the inverted domain will switch back to its initial polarization. This explains the fact that no inverted domains were observed using the $3 \mathrm{~nm}$ SWNT electrode.

Note that domain reversal can also be due to charge injection from the probe during writing. ${ }^{16}$ Upon contacting the inverted domain with a grounded tip during reading, the field between these injected charges and the probe can be large enough to switch the domain back. However, this effect does not occur for epitaxial PZT film thicknesses below 50 
$\mathrm{nm} .{ }^{16}$ The electric field at the probe-tip end during writing can also be high enough to create oxygen vacancies at the surface, which are positively charged and can neutralize the lead vacancies, thus reducing the built-in electric field. But even with a zero built-in electric field, analysis of the energetic still predicts a negative rate $(f=-4.80 \mu \mathrm{J} / \mathrm{m})$.

The energy reduction rate can be made positive by decreasing the surface area and volume over which the domain wall and depolarization forces are exerted. This can be achieved by thinning the ferroelectric film to the level where a fully inverted domain is obtained through the entire film thickness down to the grounded electrode. The energy reduction induced by the coercive electric field will then be larger than the surface energy of the domain wall, depolarization and built-in field energies. Therefore, the thinner the film thickness, i.e., the smaller the sides of domain walls, the more stable the inverted domains are. Full inversion down to the bottom electrode can also be achieved by increasing the bias pulse or by increasing the electrode size, which can create a higher electric field. In this case, however, the domain size will be larger, corresponding to lower storage densities. This is the case of the $9 \mathrm{~nm}$ SWNT electrode [Fig. $1(\mathrm{e})$ ], where stable but larger domains were written with only $7 \mathrm{~V}$ pulses. At this pulse bias, the energy reduction rate is estimated to be $0.10 \mu \mathrm{J} / \mathrm{m}$ in the lateral direction and $0.07 \mu \mathrm{J} / \mathrm{m}$ in the vertical direction. Here the domain is approximated by a prolate spheroid ${ }^{9}$ that is truncated at the end.

Thus, for sub-10 nm inverted domains to be stable in thin PZT films, inversion must occur through the entire film thickness with domain wall sides small enough for the coercive field energy to exceed the domain wall, depolarization and built-in field energies. This depends highly on the ratio of the electrode diameter to the ferroelectric film thickness [Figs. 2(c) and 2(d)]. Figure 2(e) depicts the minimum electrode size for predicted stable domain switching for various PZT thicknesses at different applied biases (properties of the $50 \mathrm{~nm}$ thick PZT film are assumed without a built-in field). For instance, the PZT film has to be thinner than $23 \mathrm{~nm}$ for a $3 \mathrm{~nm}$ electrode to write stable domains at $4 \mathrm{~V}$ bias pulses.

Our analysis is validated by using a single crystal 17nm-thick PZT film, ${ }^{13}$ which exhibits also very good butterflylike C-V and squarelike P-V hysteresis loops with high switching speeds [Figs. 3(a) and 3(b)]. The film has a built-in electric field of $2.3 \times 10^{7} \mathrm{~V} / \mathrm{m}$ and a coercive field of 3.7 $\times 10^{7} \mathrm{~V} / \mathrm{m}$. The simulated electric field mapping shows that stable domain inversion with sub-10 nm size across the entire $17 \mathrm{~nm}$ thickness can be obtained using a $3 \mathrm{~nm}$ SWNT electrode by applying only a $5 \mathrm{~V}$ pulse [Fig. 3(c)]. Figure 3(d) shows experimental PFM images of a $3 \times 1$ matrix of inverted dots written under the same conditions. Dot sizes of $4 \mathrm{~nm}$ in diameter, i.e., 10 unit-cell size, are written reliably. Such domain size corresponds to potential $40 \mathrm{Tbit} /$ in. $^{2}$ data storage densities.

Other nanopencils with individual SWNT electrodes (2-3 nm in diameter) were also used. No inverted domains were observed on the $50 \mathrm{~nm}$ thick PZT film at $10 \mathrm{~V}$ pulses. Instead of decreasing the film thickness, we increased the size of these electrodes by electrochemically reducing gold nanoparticles at the exposed tip, which allowed inverted domains to be formed as described in Ref. 13. This observation reaffirms the dependence of domain stability on the ratio of
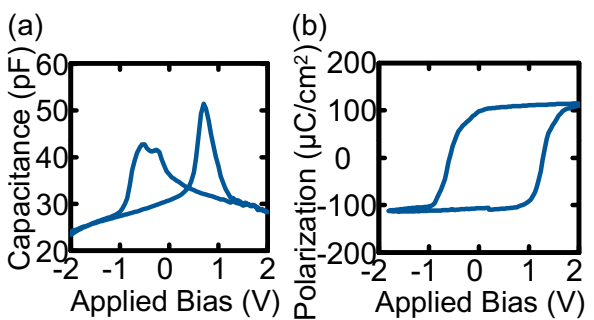

(c)

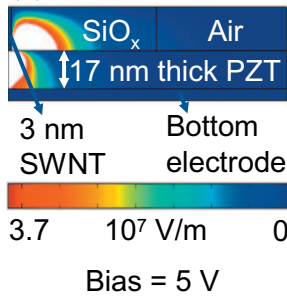

(d)

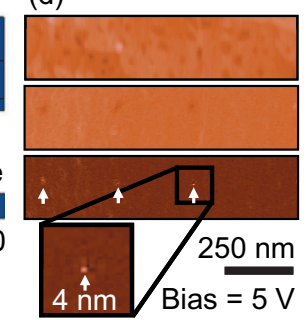

FIG. 3. (Color) [(a) and (b)] Capacitance-voltage (a) and polarizationvoltage (b) hysteresis loops. Measurements taken on a $17 \mathrm{~nm}$ single crystal PZT film. (c) Simulated cross-sectional mappings of the electric field component along the polarization axis under a $5 \mathrm{~V}$ bias with a $3 \mathrm{~nm}$ SWNT electrode. Domain inversion through the entire film thickness is predicted. (d) PFM height (top), amplitude (middle) and phase (bottom) images of the 17-nm-thick PZT-film surface with $4 \mathrm{~nm}$ ferroelectric inverted domains formed by applying $5 \mathrm{~V}$ pulses to the film through the nanopencil with $3 \mathrm{~nm}$ electrode shown in Fig. 1(b).

electrode size to the ferroelectric film thickness.

In conclusion, stable inverted domains less than $10 \mathrm{~nm}$ in diameter can be formed in ferroelectric films provided polarization inversion occurs through the entire ferroelectric film thickness. Polarization inversion depends strongly on the ratio of the electrode size to the ferroelectric film thickness.

Part of Y.Z.'s work was supported by the Office of Science, Office of Basic Energy Sciences, of the U.S. Department of Energy under Contract No. DE-AC02-05CH11231.

${ }^{1}$ C. H. Ahn, T. Tybell, L. Antognazza, K. Char, R. H. Hammond, M. R. Beasley, O. Fischer, and J. M. Triscone, Science 276, 1100 (1997).

${ }^{2}$ C. H. Ahn, M. R. Rabe, and J. M. Triscone, Science 303, 488 (2004).

${ }^{3}$ Y. Cho, S. Hashimoto, N. Odagawa, K. Tanaka, and Y. Hiranaga, Nanotechnology 17, S137 (2006).

${ }^{4}$ Y. Cho, S. Hashimoto, N. Odagawa, K. Tanaka, and Y. Hiranaga, Appl. Phys. Lett. 87, 232907 (2005).

${ }^{5}$ Y. Cho, K. Fujimoto, Y. Hiranaga, Y. Wagatsuma, A. Onoe, K. Terabe, and K. Kitamura, Nanotechnology 14, 637 (2003).

${ }^{6}$ V. Garcia, S. Fusil, K. Bouzehouane, S. Enouz-Vedrenne, N. D. Mathur, A. Barthélémy, and M. Bibes, Nature (London) 460, 81 (2009)

${ }^{7}$ N. Tayebi, N. Yoshie, R. C. Chen, P. C. Collier, K. P. Giapis, and Y. Zhang, Appl. Phys. Lett. 93, 103112 (2008).

${ }^{8}$ X. Li, A. Mamchik, and I. W. Chen, Appl. Phys. Lett. 79, 809 (2001).

${ }^{9}$ B. Wang and C. H. Woo, J. Appl. Phys. 94, 610 (2003)

${ }^{10}$ D. J. Kim, J. Y. Jo, Y. S. Kim, Y. J. Chang, J. S. Lee, J. G. Yoon, T. K. Song, and T. W. Noh, Phys. Rev. Lett. 95, 237602 (2005).

${ }^{11}$ E. Cooper, S. R. Manalis, H. Fang, H. Dai, K. Matsumoto, S. C. Minne, T. Hunt, and C. F. Quate, Appl. Phys. Lett. 75, 3566 (1999).

${ }^{12}$ H. Dai, N. Franklin, and J. Han, Appl. Phys. Lett. 73, 1508 (1998).

${ }^{13}$ See supplementary material at http://dx.doi.org/10.1063/1.3280371 for the detailed experimental methods.

${ }^{14}$ Z. Chen, Q. Tran, N. Franklin, R. J. Chen, L. P. Wang, Q. Ma, V. Rao, S. Y. Yang, R. Ramesh, T. Masuda, T. Jinbo, K. Suu, C. B. Eom, P. Zhang, and P. Coon (unpublished).

${ }^{15}$ S. Kim, N. R. Franklin, S. Y. Yang, Z. Chen, R. J. Chen, Q. Tran, S Mohammadi, Q. Ma, and V. Rao (unpublished).

${ }^{16}$ S. Bühlmann, E. Colla, and P. Muralt, Phys. Rev. B 72, 214120 (2005). 